\title{
Role of miR-134 in angiotensin II-induced vascular cell pathological changes in atherosclerosis
}

\author{
Jing Chen*, Qi Hu, Bofang Zhang, Xiaopei Liu, Shuo Yang, Hong Jiang \\ Department of Cardiology, Renmin Hospital of Wuhan University, Wuhan 430060, China \\ *For correspondence: Email: chenjingjjx@163.com; Tel: +27-88041911-81046
}

Sent for review: 27 December 2018

Revised accepted: 23 April 2019

\begin{abstract}
Purpose: To investigate the role of miR-134 in vascular smooth muscle cell dysfunction-related cardiovascular disease.

Methods: The effect of miR-134 was evaluated after human aortic smooth muscle cells (HASMCs) were transfected with miR-134 mimics. The expression levels of $p$-Akt, mechanistic target of rapamycin (mTOR), cleaved caspase-3, $p 53$, and $\beta$-actin were evaluated by immunoblotting. Terminal deoxynucleotidyl transferase dUTP nick-end labeling was used to measure cell apoptosis. Reactive oxygen species levels were assayed by fluorescence microscopy after staining with 2',7'dichlorofluorescein diacetate.

Results: Angiotensin II treatment induced miR-134 expression and Akt/mTOR activation, and inhibited cell viability in HASMCs $(p<0.01)$. Co-treatment with miRNA-134 reversed Ang Il-induced HASMC dysfunction $(p<0.01)$. Overexpression of miR-134 is protective in Ang II-induced oxidative stress and apoptosis via the Akt/mTOR pathway $(p<0.05)$.

Conclusion: MicroRNA-134 in HASMCs is a potential therapeutic target for preventing Ang II-induced cardiac dysfunction via modulating Akt/mTOR pathway.
\end{abstract}

Keywords: MiR-134, Akt/mTOR pathway, Oxidative stress, HASMCs, Atherosclerosis

\begin{abstract}
This is an Open Access article that uses a fund-ing model which does not charge readers or their institutions for access and distributed under the terms of the Creative Commons Attribution License (http://creativecommons.org/licenses/by/4.0) and the Budapest Open Access Initiative (http://www.budapestopenaccessinitiative.org/read), which permit unrestricted use, distribution, and reproduction in any medium, provided the original work is properly credited.

Tropical Journal of Pharmaceutical Research is indexed by Science Citation Index (SciSearch), Scopus, International Pharmaceutical Abstract, Chemical Abstracts, Embase, Index Copernicus, EBSCO, African Index Medicus, JournalSeek, Journal Citation Reports/Science Edition, Directory of Open Access Journals (DOAJ), African Journal Online, Bioline International, Open-J-Gate and Pharmacy Abstracts
\end{abstract}

\section{INTRODUCTION}

Atherosclerosis is a common cardiovascular disease which often leads to vascular lesions, arterial intimal thickening, increased infiltration of inflammatory cells, and the formation of a lipidrich fibrous plaques [1,2]. The presence of atherosclerosis influence coronary arteries due to the build-up of plaque. Vascular smooth muscle cell (VSMC) dysfunction plays an important role on the pathogenesis of cardiovascular disease[3].
Previous studies showed that multiple mechanisms are involved in cardiac remodeling among these, the renin-angiotensin system has been associated with the progression of cardiovascular diseases through angiotensin II (Ang II), which acts as a growth factor regulating cardiovascular homeostasis and blood volume [4]. Angiotensin II is involved in regulating migration, proliferation and hypertrophy in VSMCs [5]. Angiotensin II can also induce the activation of calcineurin and the mitochondria- 
dependent apoptotic pathway [5]. Importantly, Ang II causes rapid activation of Akt, mitogenactivated protein kinase (MAPK), MAPK/ERK kinase (MEK), and C-Jun N-terminal kinase (JNK), accompanied by cellular survival, hypertrophy, or apoptosis $[6,7]$.

Previous studies have shown that both angiotensin II and reactive oxygen species (ROS) stimulation leads to the initiation of downstream signaling events, such as activation of the Akt/mTOR pathway [8,9]. Reactive Oxygen Species generation and its associated inflammatory responses including enhanced lipid metabolism and cell death are involved in the causation of different cardiovascular diseases, including atherosclerosis. Mammalian target of rapamycin (mTOR) and Akt both improve cardiac protection in vascular disorder, hypertension, and cardiovascular abnormalities. Interestingly, recent research reported that inhibition of the Akt/mTOR pathway inhibit atherosclerosis progression and raise the stability of atherosclerotic plaques [10].

MicroRNAs are endogenous, non-coding small RNA molecules which play key roles in cell differentiation, apoptosis, and cardiovascular diseases. Some microRNAs, such as miR-134, miR-9, and miR-219 are significantly differentially expressed in coronary artery disease. For example, miR-134 serves a critical regulator in various cardio-biological processes including lipid accumulation and proinflammatory cytokine secretion. Previous research reported that miR134 is a factor related to heart failure, and serves as a biomarker for myocardial infarction [11]. However, the pathogenic mechanisms of miR134 in VSMC dysfunction-related cardiovascular disease remain unknown. Therefore, this study aims to investigate the role miR-134 in the function of vascular smooth muscle cells.

\section{EXPERIMENTAL}

\section{Cell culture and transfection}

Human aortic smooth muscle cells (HASMCs) were purchased from the American Type Culture Collection (ATCC), and were maintained in Dulbecco's Modified Eagle's Medium highglucose medium supplemented with $10 \%$ fetal bovine serum (FBS), $100 \mathrm{U} / \mathrm{ml}$ penicillin and 100 $\mathrm{mg} / \mathrm{ml}$ streptomycin at $37{ }^{\circ} \mathrm{C}$ in a humidified atmosphere of $5 \% \mathrm{CO}_{2}$. Human aortic smooth muscle cells were plated at a density of $6 \times 10^{4}$ cells per well into six-well plates. miR-134 mimics were synthesized for miR-134 overexpression and transfected into HASMCs using Lipofectamine $^{\mathrm{TM}} 2000$ (Thermo Fisher Scientific)
$48 \mathrm{~h}$ before Ang II treatment. The efficiency of transfection was evaluated using qRT-PCR and immunoblotting.

\section{Quantitative real-time PCR}

Human aortic smooth muscle cells were harvested and total RNA was isolated using the TRIzol reagent (Invitrogen), and the miR?134 level was measured by qRT-PCR using the Universal cDNA synthesis and SYBR Green Master Mix kits. The expression of miR-134 was normalized to rRNA U6. Gene expression data were analyzed using the cycle threshold method.

The sequence of the miR-134 mimic primer used in the present study was: 5'UGUGACUGGUUGACCAGAGGGG-3'.

\section{Western blotting}

Human aortic smooth muscle cells were lysed and extracted in lysis buffer. The concentration of the protein was determined using Lowry's method and the proteins were separated using $10 \%$ sodium dodecyl sulfate polyacrylamide gel electrophoresis (SDS-PAGE). The proteins were transferred to a polyvinylidene difluoride (PVDF) membrane and incubated in blocking solution (5 $\%$ non-fat milk). The membranes were then incubated with 1:1000 dilution primary antibody (anti-AKT, anti-pAKT, anti-mTOR, anti-pmTOR, anti-cleaved caspase-3, anti-p53, anti- $\beta$-actin) overnight at $4^{\circ} \mathrm{C}$. Subsequently, the membranes were incubated with secondary antibody for $1 \mathrm{~h}$ and visualized using a chemiluminescence system.

\section{Cell viability assay}

Human aortic smooth muscle cells viability was detected using a Viability Assay kit (Abcam, ab112120). HASMCs were washed with phosphate-buffered saline, then incubated in fresh medium containing dye-loading solution for $1 \mathrm{~h}$ at $37^{\circ} \mathrm{C}$. After the removal of dye-loading solution, the absorption values were measured at 405/460 nm using ELISA reader.

\section{TUNEL assay}

Terminal deoxyribonucleotidyl transferasemediated dUTP-digoxigenin nick-end labeling (TUNEL) assay was conducted following a previously described protocol [12].

\section{Monitoring ROS generation}

Dichlorofluorescein dye (non-fluorescent CMH2DCFDA), which can diffuse through the cell 
membrane, was used to evaluate ROS levels. The levels of ROS in HASMCs were determined using a fluorescence microscope with $\mathrm{CM}$ H2DCFDA dye, after incubation with the miR-134 mimic, Ang II, or Akt activator. The fluorescence of DCFDA was measured with an excitation wavelength of $488 \mathrm{~nm}$, and its emission was detected using a $520 \mathrm{~nm}$ band-pass filter.

\section{Statistical analysis}

Statistical analyses were performed using oneway analysis of variance followed by t-test for multiple comparisons, using GraphPad Prism. Data are expressed as mean \pm SEM. $p<0.05$ was considered to indicate statistically significant difference.

\section{RESULTS}

Effect of Ang II on miR-134 expression, AKT/mTOR activation, and cell viability of HASMCs

To determine whether miR-134 expression was changed with Ang II treatment in HASMCs, the expression level of miRQ134 was measured using qRT ?PCR. As shown in Figure $1 \mathrm{~A}$, Ang II drove the time-dependent decrease of miR⿴囗134 expression in HASMCs. Based on these analyses, the 48-h treatment duration with Ang II was used for subsequent experiments.

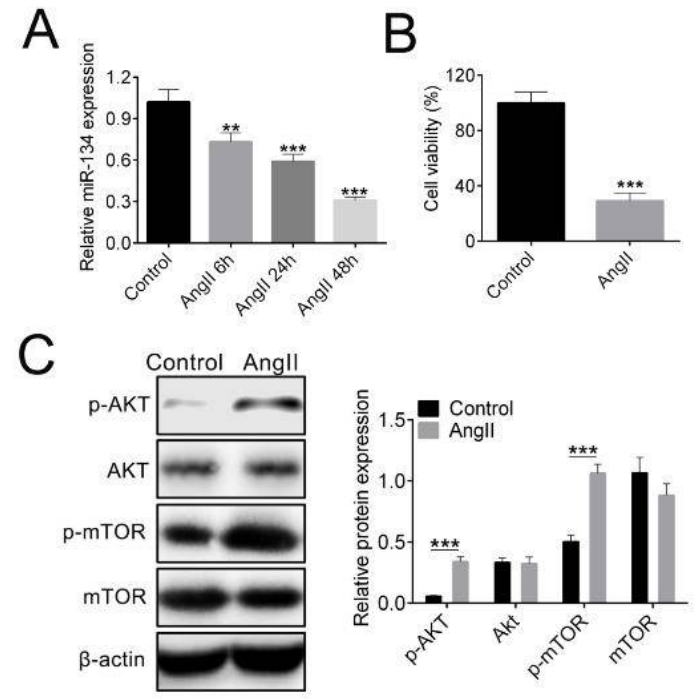

Figure 1: Effect of Ang II treatment in HASMCs. (A) Time-course of miRNA-134 expression following Ang II treatment, determined by qRT-PCR. (B) HASMC viability was assessed using Viability Assay kit. (C) The expression of indicated proteins in HASMCs treated with or without Ang II was detected by Western blot. . (Data are mean \pm SEM, Student's t-test, ${ }^{* *} p<$ $0.01,{ }^{\star \star *} p<0.005$
To assess the effect of Ang II on the activation of Akt signaling and cell apoptosis, HASMCs were treated with $100 \mathrm{nM}$ of Ang II for $48 \mathrm{~h}$. As seen in Figures $1 \mathrm{~B}$ and $\mathrm{C}$, Ang II significantly decreased cell viability $(p<0.01$; Figure $1 \mathrm{~B})$ and enhanced the activation of Akt and mTOR $(p<0.01$; Figure $1 \mathrm{C})$. These data indicate that Ang II treatment can induce AKT/mTOR activation and inhibit cell viability and miR-134 expression in HASMCs.

\section{Overexpression of miRNA-134 protect against Ang II-induced HASMC dysfunction}

To demonstrate the protective effect of miR-134 on Ang II-induced ROS generation and apoptosis, HASMCs were transfected with miR134 mimic and co-treated with Ang II. The expression of miR-134 in all groups was evaluated by quantitative RT-PCR $(p<0.01$; Figure 2 A). Intracellular ROS production was assessed following Ang II treatment using H2DCFDA ( $p<0.01$; Figure $2 \mathrm{C})$. The results are presented in Figure $2 \mathrm{C}$, and demonstrate that treatment with Ang II significantly increased the cellular oxidative stress in HASMCs. This trigger effect of Ang II was completely reversed by miR134. In addition, treatment with miR-134 mimic alone did not significantly alter ROS production. Previous studies demonstrated that Ang II induces hypertrophy, proliferation, or apoptosis in VSMCs [13-15].

Thus, to investigate whether the miR-134 is involved in the mechanism of apoptosis, HASMC apoptosis was assayed using the TUNEL assay and western blot. Angiotensin II treatment alone caused a marked increase the number of cells that were positive for TUNEL staining $(p<0.01$; Figure $2 \mathrm{C}$ ). Conversely, overexpression of miR134 markedly attenuated this effect $(p<0.01$; Figure $2 \mathrm{~B}$ ). Consistent with the results of the TUNEL assay, the expression of cleaved caspase- 3 and 053 were significantly increased in the Ang II group ( $p<0.01$; Figure $2 \mathrm{D})$. However, compared with the Ang II group, the group treated with miR-134 + Ang II showed significantly decreased expressions of cellular apoptosis biomarkers ( $p<0.01$; Figure $2 \mathrm{D})$. These results suggest that miR-134 overexpression may protect HASMCs from Ang II-induced oxidative stress and apoptosis.

\section{Upregulation of miR-134 attenuates the Ang} II-induced viability decrease in HASMCs

To explore the effects of miR-134 in Ang IIinduced HASMC viability, a cell viability assay was performed. Treatment of HASMCs with Ang II significantly suppressed cell viability $(p<0.01$; Figure 3 ), whereas overexpression of miR-134 
with Ang II treatment significantly reversed the Ang II-induced effect ( $p<0.01$; Figure 3$)$. These results showed that Ang II-inhibited cellular viability is apparently reversed upon overexpression of $\mathrm{miR}-134$.

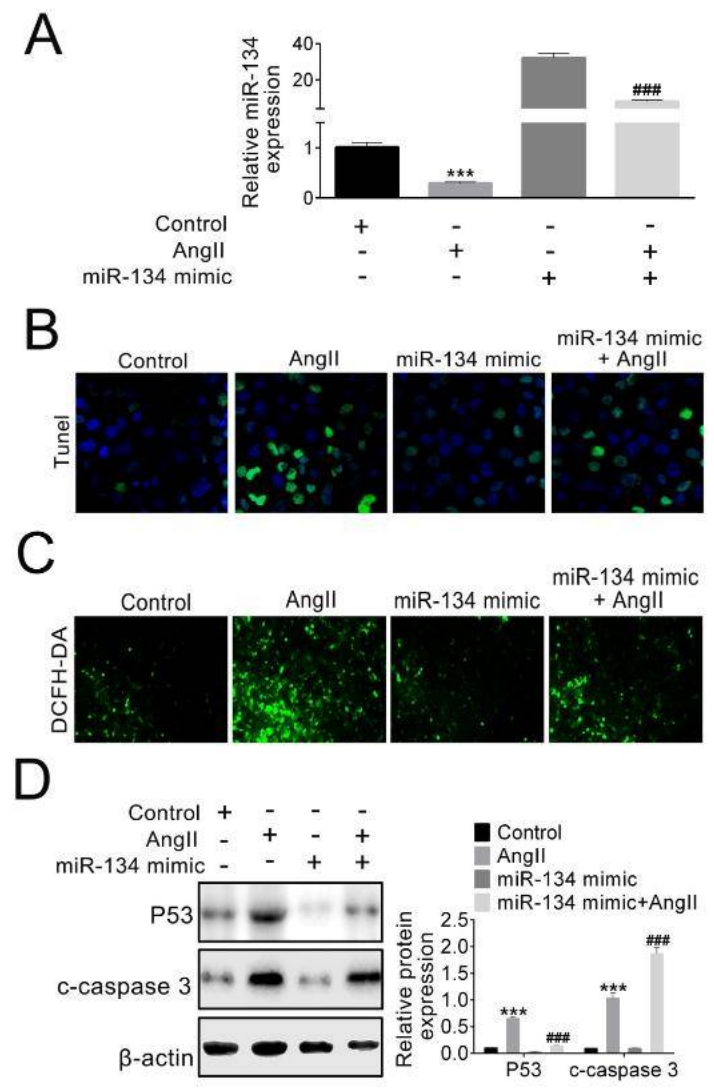

Figure 2: Effect of miR-134 in Ang II-induced cellular apoptosis and oxidative stress. (A) The expression level miR-134 after transfection with miR-134 mimic or Ang II treatment, determined by qRT-PCR. (B) Cell apoptosis was determined by TUNEL assay. (C) Cells were stained with oxidized dye to measure intracellular ROS, and then detected by DCF-derived fluorescence. (D) The expression of indicated proteins was detected by Western blot. (Data are mean \pm SEM, Student's ttest, $\# \# p<0.01$ vs Ang II, ${ }^{* \star *} p<0.005$ vs control).

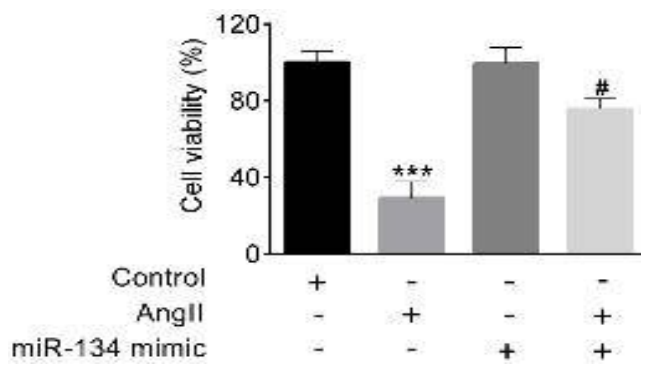

Figure 3: Effect of miR-134 on Ang II-induced viability inhibition. (A) miR-134 overexpression against Ang IIinduced injury was determined by viability assay. (Data are mean \pm SEM, Student's t-test, $\# p<0.05$ vs Ang II, ${ }^{* * *} p<0.005$

\section{MiR-134 suppresses Ang II-induced cardiac dysfunction via Akt/mTOR pathway}

To investigate the molecular mechanism of miR134134 in protecting Ang II-mediated cardiac dysfunction, this study determined whether the protective effect of miR-134 in Ang II treatment is mediated through the Akt/mTOR pathway. Cells exhibiting Ang II-induced cardiac dysfunction were pretreated with the Akt activator SC79 and pre-transfected with miR-134. Since miR-134 has anti-apoptotic and antioxidant effects, this study then assessed whether miR-134 affects the Akt activation induced by Ang II.

Western blot showed that miR-134 significantly inhibited the Ang II-induced activation of Akt and $\operatorname{mTOR}(p<0.05$; Figure 4C). Thus, overexpression of miR-134 attenuate Ang IIinduced Akt/mTOR pathway activation. To ascertain whether Ang II-induced Akt activation and cardiomyocyte dysfunction is associated with cellular ROS generation, this study first analyzed the effect of Akt activation on Ang II-induced increases in intracellular ROS production in HASMCs, using DCFDA staining. As shown in Figure 4A, Ang II treatment significantly increased ROS production when compared with the control and miR-134 only groups, whereas miR-134 + Ang II treatment markedly attenuated these effects. Conversely, combined treatment with miR-134 + SC79 suppressed the protective effects of miR-134 ( $p<0.05$; Figure 4A). On the other hand, compared with the miR-134 + Ang II group, the cell apoptosis, as measured by TUNEL staining and the expression levels of cleaved caspase-3 and p53, were significantly increased upon miR-134 + SC79 treatment $(p<$ 0.05 ; Figure 4B \& $\mathrm{C}$ ). These results indicate that miR-134 provides a protective mechanism in Ang II-induced cardiac dysfunction via the Akt/mTOR pathway.

\section{DISCUSSION}

There are multiple physiological and pathological processes involved in atherosclerosis, such as foam cell formation, activation of the inflammatory response, and ROS generation [16]. A large body of clinical and laboratory data are now available confirming that that the reninangiotensin system is mechanistically relevant in the pathogenesis of atherosclerosis [4,17]. Renin-angiotensin system has been well established as a major physiological system that regulates blood pressure, sodium balance, and cardiovascular status, and is involved in atherosclerosis development and vascular $\mathrm{NAD}(\mathrm{P}) \mathrm{H}$ oxidase activation [17]. 

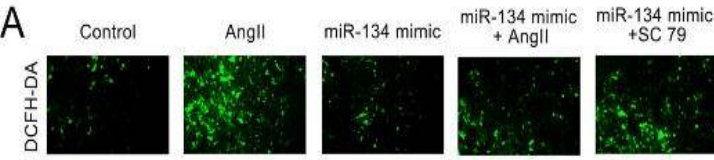

B
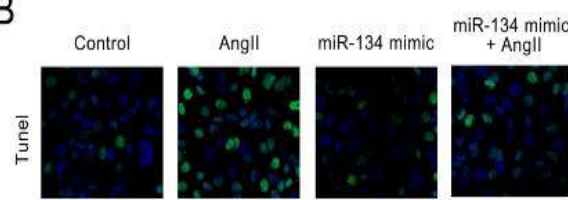

C

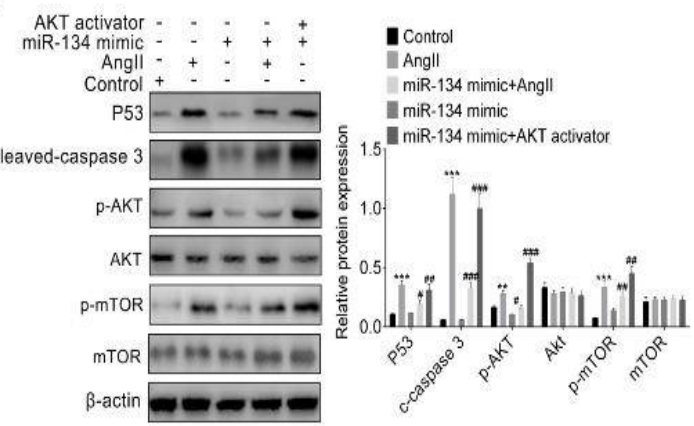

Figure 4: miR-134 modulate Ang II-induced cell dysfunction in HASMCs via the Akt/mTOR signaling pathway. (A) Cells were stained with oxidized dyes to measure intracellular ROS, which was then detected by DCF-derived fluorescence. (B) Apoptosis rate was determined by the TUNEL assay. (C) The expression of indicated proteins was detected by Western blot. (data are mean \pm SEM, Student's t-test, \#\# $p<0.01$ vs Ang II, ${ }^{* * *} p<0.005$

Activation of the renin-angiotensin system accelerates HASMC hypertrophy and inhibits cell viability, thereby contributing to cardiovascular disease. $[18,19]$. Recent research has reported that Ang II augments proinflammatory activity in the vascular wall byinducing ROS generation and inflammatory cytokines. However, the signaling pathway involved in regulating the cell viability and apoptosis of HASMCs in atherosclerosis remains unclear.

In the present study, this study first determined that the overexpression of miR-134 could attenuate Ang II-induced oxidative stress and apoptosis in HASMCs. These results revealed that the expression level of miR-134 was significantly decreased after Ang II treatment, compared with the untreated group. This finding was supported by the results of quantitative RTPCR. Furthermore, Ang II significantly decreased cell viability and enhanced the activation of Akt and mTOR. These data are consistent with recent findings that Ang II activates the Akt/mTOR pathway through the small GTPase Rac1 and a NAD(P)H oxidase $[20,21]$. Activation of the Akt/mTOR pathway is important for the regulation of metabolism and proliferation in HASMCs.
Recent studies showed that the Akt/mTOR pathway is a target of ROS, and ROS play a role as second messengers mediating the trigger effect of Ang II [22]. MicroRNA-134, located on 14q32.31, has been reported to regulate the pathological processes of various vascular diseases [23,24]. Considering that Akt/mTOR pathway activation and oxidative stress and miRNA-134 are important factors inthe process of cardiovascular disease, this study analyzed the functional relevance of the $A \mathrm{kt} / \mathrm{mTOR}$ pathway, oxidative stress, and miR-134 in Ang IIinduced HASMC dysfunction.

In this study, the Ang II treatment model in HASMCs was used to mimic the characteristic vascular pathologies of atherosclerosis in vitro. In the course of atherosclerotic disease, the balance between cell apoptosis and survival of VSMCs is associated with atherosclerotic plaque instability or stability [25]. Although Ang II could stimulate HASMC proliferation, a previous study showed that long-term treatment with Ang II may induce cell apoptosis. The effects of miR-134 on Ang II-induced cell apoptosis was found.

A previous research has shown that the protective effect of AKT/mTOR pathway could modulate intracellular ROS generation and regulate vascular smooth muscle dysfunction [26]. This study found that the overexpression of miR-134 significantly increased the expressions of cellular antioxidant factors, thereby protecting HASMCs from Ang II-induced cardiac dysfunction via the Akt/mTOR pathway. These findings identified miR-134 as a potential therapeutic target in preventing Ang II-induced cardiac dysfunction.

\section{CONCLUSION}

These results demonstrate that Ang II induces cellular apoptosis and increased oxidative stress. The expression of miR-134 in HASMCs will be critical for cardiac therapeutic applications. microRNA-134 provides a protective mechanism in Ang II-induced cardiac dysfunction via the Akt/mTOR pathway. This study reveals that miR134 exerts a protective effect in cardiac dysfunction and also provides an alternative therapeutic approach for atherosclerosis.

\section{DECLARATIONS}

\section{Conflict of interest}

No conflict of interest is associated with this work. 


\section{Contribution of authors}

We declare that this work was done by the researchers listed in this article. All liabilities related with the content of this article will be borne by the authors. JC and $\mathrm{QH}$ designed all the experiments and revised the paper. $\mathrm{BZ}$ and $\mathrm{XL}$ formed the experiments, SY and HJ wrote the paper.

\section{Open Access}

This is an Open Access article that uses a funding model which does not charge readers or their institutions for access and distributed under the terms of the Creative Commons Attribution License (http://creativecommons.org/licenses/by/ 4.0) and the Budapest Open Access Initiative (http://www.budapestopenaccessinitiative.org/rea d), which permit unrestricted use, distribution, and reproduction in any medium, provided the original work is properly credited.

\section{REFERENCES}

1. Libby $P$, Ridker $P M$, Maseri $A$. Inflammation and atherosclerosis. Circulation. 2002; 105(9): 1135-1143.

2. Shai S-Y, Sukhanov S, Higashi $Y$, Vaughn C, Kelly J, Delafontaine P. Smooth muscle cell-specific insulin-like growth factor-1 overexpression in Apoe mice does not alter atherosclerotic plaque burden but increases features of plaque stability. Arteriosclerosis, thrombosis, and vascular biology. 2010; 30(10): 1916-1924.

3. Shi N, Chen $S-Y$. Mechanisms simultaneously regulate smooth muscle proliferation and differentiation. Journal of biomedical research. 2014; 28(1): 40.

4. Schmieder RE, Hilgers KF, Schlaich MP, Schmidt BM. Renin-angiotensin system and cardiovascular risk. The Lancet. 2007; 369(9568): 1208-1219.

5. Li L, Gao P, Zhang H, Chen H, Zheng W, Lv X, Xu T, Wei $Y$, Liu D, Liang C. SIRT1 inhibits angiotensin II-induced vascular smooth muscle cell hypertrophy. Acta Biochim Biophys Sin. 2010; 43(2): 103-109.

6. Ushio-Fukai M, Alexander RW, Akers M, Yin Q, Fujio Y, Walsh $K$, Griendling KK. Reactive oxygen species mediate the activation of Akt/protein kinase $B$ by angiotensin II in vascular smooth muscle cells. Journal of Biological Chemistry. 1999; 274(32): 22699-22704.

7. Eguchi S, Dempsey PJ, Frank GD, Motley ED, Inagami T. Activation of MAPKs by angiotensin II in vascular smooth muscle cells metalloprotease-dependent EGF receptor activation is required for activation of ERK and p38 MAPK but not for JNK. Journal of Biological Chemistry. 2001; 276(11): 7957-7962.

8. Han X, Sun S, Zhao M, Cheng X, Chen G, Lin S, Guan Y, $Y u X$. Celastrol stimulates hypoxia-inducible factor-1 activity in tumor cells by initiating the ROS/Akt/p70S6K signaling pathway and enhancing hypoxia-inducible factor-1a protein synthesis. PLoS One. 2014; 9(11): e112470.

9. Wan J, Liu T, Mei L, Li J, Gong K, Yu C, Li W. Synergistic antitumour activity of sorafenib in combination with tetrandrine is mediated by reactive oxygen species (ROS)/Akt signaling. British journal of cancer. 2013; 109(2): 342.

10. Zhai C, Cheng J, Mujahid H, Wang H, Kong J, Yin Y, Li J, Zhang $Y$, Ji $X$, Chen $W$. Selective inhibition of $P I 3 K / A k t / m T O R$ signaling pathway regulates autophagy of macrophage and vulnerability of atherosclerotic plaque. PloS one. 2014; 9(3): e90563.

11. Hoekstra M, van der Lans CA, Halvorsen B, Gullestad $L$, Kuiper J, Aukrust P, van Berkel TJ, Biessen EA. The peripheral blood mononuclear cell microRNA signature of coronary artery disease. Biochemical and biophysical research communications. 2010; 394(3): 792-797.

12. Son B-K, Kozaki K, lijima K, Eto M, Nakano T, Akishita $M$, Ouchi Y. Gas6/AxI-PI3K/Akt pathway plays a central role in the effect of statins on inorganic phosphateinduced calcification of vascular smooth muscle cells. European journal of pharmacology. 2007; 556(1-3): 1-8.

13. Ruiz-Ortega $M$, Ruperez $M$, Esteban V, Egido J. Molecular mechanisms of angiotensin II-induced vascular injury. Curr Hypertens Rep. 2003; 5(1): 73-79.

14. Dimmeler S, Zeiher AM. Reactive oxygen species and vascular cell apoptosis in response to angiotensin II and pro-atherosclerotic factors. Regulatory Peptides. 2000; 90(1-3): 19-25.

15. Mehta PK, Griendling KK. Angiotensin II cell signaling: physiological and pathological effects in the cardiovascular system. American Journal of PhysiologyCell Physiology. 2007; 292(1): C82-C97.

16. Lundqvist A, Magnusson LU, Ullström C, Krasilnikova J, Telysheva G, Dizhbite T, Hultén LM. Oregonin reduces lipid accumulation and proinflammatory responses in primary human macrophages. Biochemical and biophysical research communications. 2015; 458(3): 693-699.

17. Husain K, Hernandez W, Ansari RA, Ferder $L$. Inflammation, oxidative stress and renin angiotensin system in atherosclerosis. World journal of biological chemistry. 2015; 6(3): 209.

18. Rossi F, Castelli A, Bianco MJ, Bertone C, Brama M, Santiemma $V$. Ghrelin inhibits contraction and proliferation of human aortic smooth muscle cells by cAMP/PKA pathway activation. Atherosclerosis. 2009; 203(1): 97-104

19. Uraoka M, Ikeda K, Nakagawa Y, Koide M, Akakabe Y, Nakano-Kurimoto R, Takahashi T, Matoba S, Yamada $H$, Okigaki $M$. Prorenin induces ERK activation in endothelial cells to enhance neovascularization independently of the renin-angiotensin system. Biochemical and biophysical research communications. 2009; 390(4): 1202-1207.

20. Frey RS, Ushio-Fukai M, Malik AB. NADPH oxidasedependent signaling in endothelial cells: role in 
physiology and pathophysiology. Antioxidants \& redox signaling. 2009; 11(4): 791-810.

21. Kim J, Shao Y, Kim SY, Kim S, Song HK, Jeon JH, Suh HW, Chung JW, Yoon SR, Kim YS. Hypoxia-induced IL18 increases hypoxia-inducible factor-1a expression through a Rac1-dependent NF-KB pathway. Molecular biology of the cell. 2008; 19(2): 433-444.

22. GORIN $Y$, KIM N-H, FELIERS D, BHANDARI B, CHOUDHURY GG, ABBOUD HE. Angiotensin II activates Akt/protein kinase $B$ by an arachidonic acid/redox-dependent pathway and independent of phosphoinositide 3-kinase. The FASEB Journal. 2001; 15(11): 1909-1920.

23. Thomas RA, Scicchitano MS, Mirabile RC, Chau NT, Frazier KS, Thomas HC. MicroRNA changes in rat mesentery and serum associated with drug-induced vascular injury. Toxicology and applied pharmacology. 2012; 262(3): 310-320.
24. Lan G, Xie W, Li L, Zhang M, Liu D, Tan Y-L, Cheng H-P, Gong $D$, Huang $C$, Zheng $X-L$. MicroRNA-134 actives lipoprotein lipase-mediated lipid accumulation and inflammatory response by targeting angiopoietin-like 4 in THP-1 macrophages. Biochemical and biophysical research communications. 2016; 472(3): 410-417.

25. Ambrose JA, D'Agate DJ. Classification of systemic therapies for potential stabilization of the vulnerable plaque to prevent acute myocardial infarction. The American journal of cardiology. 2005; 95(3): 379-382.

26. Tang $Y$, Jacobi A, Vater $C$, Zou X, Stiehler M. Salvianolic acid $B$ protects human endothelial progenitor cells against oxidative stress-mediated dysfunction by modulating Akt/mTOR/4EBP1, p38 MAPK/ATF2, and ERK1/2 signaling pathways. Biochemical pharmacology. 2014; 90(1): 34-49. 